http://dx.doi.org/10.18778/2196-8403.2016.07

\author{
ATTILA MÉSZÁROS
}

\title{
„Wir schaffen das, wenn...." Anspielungen in Artikelüberschriften am Beispiel der deutschen Einwanderungsdebatte
}

Tematem artykułu jest analiza roli aluzji w debacie dotyczącej imigracji, toczącej się na łamach niemieckich czasopism. W części teoretycznej zostanie przedstawione pojęcie aluzji nie tylko jako oczywiste odniesienie do innego tekstu, ale także jako środek służący zbudowaniu interakcji pomiędzy autorem a czytelnikiem. W części empirycznej, na podstawie konkretnych przykładów, stworzona zostanie typologia, która klasyfikuje aluzję według jej źródła oraz rodzaju odniesienia do tekstu pierwotnego.

Der vorliegende Beitrag berichtet über die Rolle von Anspielungen in Artikelüberschriften am Beispiel des deutschen Einwanderungsdiskurses. Im theoretischen Teil werden Anspielungen als ein Phänomen dargestellt, das nicht nur als bloße Manifestation von Intertextualität, sondern vielmehr als kreatives Mittel in einem interaktiven Prozess zwischen Autor/in und Leser/in fungiert. Im empirischen Teil wird anhand konkreter Beispiele eine Typologie erstellt, die Anspielungen nach dem Quellenbereich und nach Art der Bezugnahme auf den Prätext klassifiziert.

This paper examines the use of allusions in article headlines, using the example of the coverage of German immigration. In the theoretical section, allusions are presented as a phenomenon that functions not only as a manifestation of intertextuality, but rather as a creative instrument in an interactive process between the author and the reader. In the empirical section, a typology is created by means of concrete examples, which classifies allusions according to the source range and by way of references to the 'urtext'. The analysis results are interpreted and conclusions are drawn. 


\section{Einleitung}

Der 2011 ausgebrochene Bürgerkrieg in Syrien gehört von Anfang an zu den zentralen Themen der Presse. Der Waffenkonflikt ist trotz kontinuierlicher Verhandlungen zwischen den Gegnerparteien bis heute ungelöst und resultierte u.a. in der seit 2015 andauernden Flüchtlingskrise, die insbesondere für die Bevölkerung Europas einen mehrfachen Schock darstellt. Es handelt sich nämlich um eine humanitäre Katastrophe, die nicht in einem entfernten, unbekannten Land stattfindet, sondern in Form der fliehenden Massen manche europäischen Länder direkt betrifft. Somit gilt es als ein Phänomen, über welches man nicht ausschließlich aus der - teilweise womöglich auch gefilterten oder manipulierten - Berichterstattung der Presse erfährt, sondern welches man in vielen Orten auch direkt miterlebt.

Die Relevanz der oben geschilderten Flüchtlingskrise drückt sich u.a. in ihrer Medialisierung aus. Sowohl überregionale und führende Presseprodukte als auch örtliche Medien beschäftigen sich spätestens seit 2015 mehr oder weniger regelmäßig mit dem Thema Flüchtlinge. Das gilt exponentiell in jenen Ländern, die von der Flüchtlingskrise direkt betroffen sind, so z. B. in Ungarn, Österreich und vor allem in Deutschland. Der Verfasser dieses Beitrags arbeitet seit Anfang 2016 an einem diskurslinguistisch orientierten Forschungsprojekt, das auf die Darstellung der Flüchtlingskrise im Laufe des Jahres 2015 in der deutschen, ungarischen und slowakischen Presse gerichtet ist. Zu den Zielsetzungen dieses Projekts gehören u.a. die Ermittlung des diskursspezifischen Vokabulars sowie jener Sprachgebrauchsmuster, die die thematisierte Flüchtlingsdebatte in den untersuchten Sprachen ausmachen ${ }^{1}$.

1 Der vorliegende Beitrag ist zwar im Rahmen einer diskurslinguistisch orientierten Untersuchung entstanden, auf theoretische und methodologische Fragen der Diskursanalyse wird hier nur sehr begrenzt eingegangen, u.a. auch aus dem Grunde, dass selbst der Diskursbegriff - trotz der umfangreichen Fachliteratur - bis heute sehr umstritten ist. Der Verfasser vertritt die Ansicht, dass eine Präzisierung des Terminus nur dann möglich ist, wenn die Akzentuierungen und Ausrichtungen dem konkreten Forschungsinteresse entsprechend festgelegt werden. Hier werden unter Diskurs in Anlehnung an ADAMZIK (2004:254) thematisch zusammenhängende Aussagekomplexe verstanden, die in Form von (Text)Korpora aufbereitet und für linguistische Untersuchungen verfügbar gemacht werden können. Einen umfassenden Überblick zum Begriff ,Diskurs ${ }^{e}$ und dessen möglichen Interpretationen liefert GARDT (2007), zu den einzelnen Strömungen der Diskursanalyse siehe NIEHR (2014a). 
Das Sprachmaterial für die Analysen wurde in Form von Korpora aufbereitet. In diese wurden Artikel aus den Zeitungen Frankfurter Allgemeine Zeitung und Süddeutsche Zeitung aufgenommen. Beide gehören zu den führenden meinungsbildenden Printmedien Deutschlands und auch in Hinblick auf linguistische Analysen liefern beide Quellen relevantes und qualitatives Material. Die Kandidaten für die Aufnahme ins Korpus wurden in zwei Schritten ausgewählt. Es wurde zuerst eine Recherche durchgeführt, wo jene Artikel, die den Terminus ,Flüchtling' enthalten, aussortiert wurden. Im zweiten Schritt wurden die so ermittelten Texte einzeln analysiert; das Hauptinteresse galt dabei deren Überschriften. Es war auffallend, dass während in einigen Presseprodukten fast ausschließlich eindeutige, zum anschließenden Haupttext in direkter Beziehung stehende Titel verwendet wurden, in anderen Zeitungen besonders häufig mit anspielungshaften Konstruktionen operiert wurde. Diese Beobachtung gab den Anstoß, eine Analyse der Überschriften vor dem Hintergrund der aktuellen Einwanderungsdebatte durchzuführen.

Im vorliegenden Beitrag wird versucht, in dem oben thematisierten Kontext Anspielungen als ein Phänomen darzustellen, das nicht nur als bloße Manifestation von Intertextualität, sondern vielmehr als kreatives Mittel in einem interaktiven Prozess zwischen Autor/in und Leser/in zu betrachten ist. Sie treten dabei als Instrumente mit doppelter Funktion auf: Einerseits zeichnen sie sich als besonders kreative Mittel im sog. journalistischen Kanon aus, indem sie den Ko-Text, d.h. den eigentlichen Artikel vermarkten. Auf der anderen Seite erfordern sie von den Leser/innen ein umfangreiches kulturelles Wissen, mit dessen Hilfe es erst möglich ist, die Absicht der Autor/innen bzw. den Textinhalt zu dekodieren.

\section{Gegenstand und Fragestellung}

Die Einwanderungsdebatte in Deutschland gilt in der einschlägigen Fachliteratur als bereits ausführlich erforschtes Gebiet. Diese wird thematisiert u.a. bei Jung / NiEHR / BöKE / DORENBECK (2000), NiEHR / BöKE (2000) und Wengeler (2003). Im Fokus liegt dabei die diskurslinguistische Perspektive im besonderen Hinblick auf die Wort- bzw. Argumentationsebene. Diese sowie einige frühere Forschungen (vgl. u.a. KLEIN 1989, BÖKE / LIEDTKE / WENGELER 1996, NIEHR 2014b), die auf einzelne Aspekte im politischen Sprachgebrauch gerichtet waren, galten als Vorreiter der heute bereits selbstständig behandelten sprachwissenschaftlichen Disziplin, der Politolinguistik. 
Relativ wenig Beachtung wurde dabei der Rolle der Bildhaftigkeit in der politischen Sprache geschenkt, obwohl gerade hier Metaphern und weitere tropische Konstruktionen besonders häufig vorkommen (vgl. SvENSSON 1984:26f.). Als Wegweiser in dieser Richtung können die Untersuchungen von BöKE (1997; 2002) betrachtet werden, die die Metaphorik am Beispiel der Einwanderungsdebatte fokussieren.

Im vorliegenden Beitrag gilt das Forschungsinteresse den intertextuellen Bezügen, die durch Anspielungen in Artikelüberschriften hergestellt werden. Es wird von der Annahme ausgegangen, dass Anspielungen ebenso zum Inventar der politischen Sprache gehören und bewusst eingesetzt werden. Anhand von Beispielen aus einem nicht-repräsentativen Textkorpus wird hier auf die Fragen eingegangen, (a) in welchen Konfigurationen Anspielungen im politischen Sprachgebrauch vorkommen und (b) inwieweit der Kontext - hier der Migrationsdiskurs - den Einsatz konkreter Anspielungen bestimmt.

\section{Zur Rolle von Überschriften in Zeitungsartikeln}

Im Folgenden vertritt der Autor die Ansicht, dass die Überschriften, die als relativ selbstständige ,Kleintexte ${ }^{`}$ vor dem Haupttext nicht nur Aufmerksamkeit erregen, sondern auch eine direkte Bindung an den Textinhalt herstellen sollen, nur begrenzt als Anspielungen fungieren. Es kommt ihnen dabei mehrfach eine entscheidende Rolle zu. Sie erfüllen eine Motivations- und Selektionsfunktion, indem die Überschrift jenes Element eines Zeitungsartikels ist, anhand dessen die Leser/innen die Entscheidung treffen, den jeweiligen Text überhaupt zu lesen (REITER 2006:8). Aus diesem Grunde ist hier besondere sprachliche Kreativität gefragt, um diesen Leseanreiz zu schaffen und schließlich das (Presse)Produkt zu verkaufen. Diese Forderung wird durch das Kriterium der Sprachökonomie ergänzt, das einerseits durch die begrenzten Möglichkeiten des Zeitungslayouts, andererseits durch die Lesergewohnheiten begründet ist. Kurze Überschriften passen nicht nur technisch besser in das Layout, sondern ermöglichen den Leser/innen, anhand des Titels Informationen über den Textinhalt schneller zu erfahren (LEIDECKER 2015:196).

Diese Sprachökonomie unterstützt der Einsatz bildhafter sprachlicher Konstruktionen auch in der Pressesprache. Man versucht, die außersprachliche Wirklichkeit mit Hilfe der Sprache zu erfassen und auf diese Weise ein sog. Weltwissen zu konstruieren. Spätestens seit dem Erschienen des Buches Leben in Metaphern von LAKOFF und JOHNSON im Jahre 1980 wurde sowohl in der 
Wissenschaft als auch in der Öffentlichkeit akzeptiert, dass die menschliche Erkenntnis in großem Maße auf spielerische Art, u.a. mit Hilfe der Metaphorik erfolgt (vgl. LAKOFF/ JOHNSON 2007:11-14). Neues Wissen kann auf der Basis von bereits existierenden Wissensbeständen besser aufgebaut und fixiert werden. Da die Metaphorik genau auf diesem Prinzip fungiert (d.h. Übertragung von Attributen des bereits Bekannten auf das Neue), zeichnen sich Metaphern nicht nur als stilistische Mittel der poetischen Sprache aus, sondern als grundlegendes wissensstiftendes Instrument der Alltagssprache. Das bedeutet zugleich, dass dieses kognitive Instrumentarium - somit auch Anspielungen je nach Kommunikationsstrategie gezielt eingesetzt werden kann, um bestimmte Aspekte der $\mathrm{zu}$ vermittelnden Inhalte $\mathrm{zu}$ beleuchten und andere wiederum im Hintergrund zu lassen (NIEHR 2014b:144).

\section{Methodisches Vorgehen}

Den empirischen Analysen liegt ein theoretischer Rahmen zugrunde, der Anspielungen als Manifestierung von Intertextualität $\mathrm{zu}$ erklären versucht. Anspielungen werden demnach als sprachliche Konstrukte betrachtet, die als stark relationale Phänomene ähnlich zu den Metaphern unterschiedliche Domänen verbinden und deren Verständnis ein entsprechendes Wissensniveau bzw. Mitwirken der Kommunikationsteilnehmer/innen, also der Leser/innen erfordert. Vor diesem Hintergrund wurden die Belege darauf analysiert, inwieweit die einzelnen Artikelüberschriften als Anspielungen betrachtet werden können. Als Ausgangsbasis für weitere Analysen wurde mit der Software DNA (vgl. LEIFELD 2009:391-404) ein Textkorpus erstellt; die als Anspielung markierten Überschriften wurden dabei durch gezielte Recherchen im Internet überprüft und verifiziert. Im empirischen Teil des Beitrags werden einige Fallbeispiele ausgewählt, anhand derer die intertextuellen Beziehungen zwischen Anspielung und Prätext veranschaulicht werden.

\section{Datengrundlage}

Die hier präsentierten Ergebnisse basieren auf Untersuchungen eines Korpus zweier deutscher Tageszeitungen (s. Abs. 1). Der Untersuchungszeitraum beschränkt sich auf eine 18-monatige Periode zwischen dem 1. Januar 2015 und dem 31. August 2016. Der Grund für die Wahl dieses Zeitraumes liegt darin, dass die durch den Bürgerkrieg in Syrien generierte Flüchtlingswelle 2015 ein 
kritisches Ausmaß erreichte, wodurch in den meisten europäischen Ländern erneut eine Diskussion über die Einwanderung einsetzte. Deutschland war in dieser Situation zweifach betroffen. Einerseits musste es als Zielland der Flüchtlinge mit allen - gesellschaftlichen, wirtschaftlichen und politischen Folgen zurechtkommen. Auf der anderen Seite musste Deutschland als führende Kraft der Europäischen Union auftreten und auf transnationaler Ebene Lösungen finden oder zumindest vorstellen. Dies ergibt jedoch nicht nur einen besonders lebhaften Diskurs über die Flüchtlinge und somit ausreichendes Material für diskurslinguistische Analysen, sondern hat auch ernste und vorerst kaum überschaubare politische Konsequenzen, u.a. in Form des raschen Aufkommens populistischer bzw. radikaler Parteien in und außerhalb der Bundesrepublik. In die Analyse wurden lediglich Artikel einbezogen, die zum Ressort Politik gehören; Texte aus Sparten, wie Wirtschaft, Sport oder Kultur, bleiben hier unberücksichtigt.

Das Korpus enthält insgesamt 64 Einträge und erhebt somit keinen Anspruch auf Repräsentativität. Im Vordergrund steht vielmehr das exemplarische Aufzeigen von Anspielungen, die vor dem Hintergrund dieses spezifischen Diskurses in Überschriften von Zeitungsartikeln vorkommen. Nicht untersucht werden Anspielungen, die in den jeweiligen Ko-Text eingebettet sind. Die ermittelten Formulierungen werden typologisiert. Anhand ausgewählter Beispiele wird ausführlich dargestellt, in welchen Zusammenhängen Anspielungen entstehen.

Als Referenz dient die von WILSS (1989:73-89) aufgestellte Typologie von Anspielungen. Als Kriterium der Klassifizierung wird dabei der Quellenbereich der Prätexte verwendet. Hier werden neben generellen Domänen, wie Geschichte, Kunst oder Sport, auch einige ganz spezifische Bereiche, wie Werbung oder die Bibel, aufgeführt. Es ist anzumerken, dass die Referenztypologie von WILSS auf einem Korpus von Zeitungsartikeln basiert, das Einträge ohne Berücksichtigung ihrer Ressortzugehörigkeit enthält und Anspielungen sowohl in Überschriften als auch im Fließtext in die Analyse einbezogen werden.

\section{Anspielungen: eine Manifestierung von Intertextualität}

Anspielungen sind Phänomene, die ,sich immer auf etwas textuell schon Vorhandenes beziehen“ (WILSS 1989:3). Sie entstehen lediglich in einem Abhängigkeitsverhältnis, d.h., ihre Existenz beschränkt sich auf die Beziehungen zwischen zwei oder mehreren Texten. Somit ist es kein Zufall, dass sie in der 
Fachliteratur primär im Rahmen der Intertextualität behandelt werden (vgl. u.a. Broich / PFISTER 1985, Wilss 1989, Holthuis 1993, HeumanN 2015).

Obwohl der Begriff der Intertextualität seit seiner Einführung in die Literaturwissenschaft durch KRISTEVA (1969) so manche (Neu)Interpretationen erlebte, handelt es sich hierbei grundsätzlich um eine referentielle Relation zwischen zwei Texten, „wenn der eine Text auf irgendeine Weise auf den anderen referiert“ (PETÖFI/ OLIVI 1988: 336). BARTHES definiert Text als „ein Gewebe von Zitaten aus unterschiedlichen Stätten der Kultur" (BARTHES 2000: 190). Jeder Text gilt daher als Intertext (vgl. auch BARTHES 1981:39), als eine Art virtueller Raum, in dem textuelle Kommunikation erfolgt. Hierbei ist jeder Text als Reaktion auf ein vorausgegangenes textuelles Konstrukt und zugleich als Auslöser für zukünftige Reaktionen zu betrachten.

Intertextualität [..] setzt also das Gelingen eines ganz bestimmten Kommunikationsprozesses voraus, bei dem nicht nur Autor und Leser sich der Intertextualität eines Textes bewusst sind, sondern bei dem jeder der beiden Partner des Kommunikationsvorgangs darüber hinaus auch das Intertextualitätsbewusstsein seines Partners miteinkalkuliert.

Daraus resultiert die Frage, ob Intertextualität per se existiert, d.h. durch die absichtliche Einbettung von Fremdtexten in den eigenen Text, oder erst im Leseprozess zustande kommt, wenn diese Beziehung von Leser/innen erkannt wird. Es zeigt sich daher als nützlich, die Perspektive der Verfasser/innen und Leser/innen voneinander getrennt zu untersuchen. Da hier die Betonung auf den Anspielungen liegt, wird auf die Problematik der Intertextualität in Hinsicht auf die Produzent-Rezipient-Beziehung nur begrenzt eingegangen.

Um das Phänomen der Anspielung in dem breiten Feld der Intertextualität zu positionieren, wurden in der einschlägigen Fachliteratur bereits mehrere Versuche unternommen. PFISTER (1985:25-28) führt qualitative und quantitative Kriterien auf, um intertextuelle Bezüge zu erfassen und einzuordnen. Unter den qualitativen Kriterien werden u.a. die Referentialität, Kommunikativität und Selektivität aufgeführt. Diese versuchen eine Art Graduierung intertextueller Beziehungen auszudrücken. In diesem Sinne weisen die größte Intensivität an Intertextualität Zitate auf, da durch den präzisen Bezug auf den Prätext die Referentialität gerade bei diesen Texten am größten ist. Anspielungen gelten hingegen als weniger intertextuell, da hier die jeweiligen Prätexte in der Regel nur beiläufig oder latent erscheinen und gleichzeitig der aktiven Teilnahme der Leser/innen bei der Dekodierung der Botschaft eine größere Rolle zukommt. Diese Intensivität erscheint auch explizit in der Markierung von 
Intertextualität. HOLTHUIS (1993:91) unterscheidet dabei zwischen Globalstrategien der Referenz, die dementsprechend aus unterschiedlichen Markierungen resultieren. Zitate zeichnen sich demnach als Referenzen auf der Oberfläche aus. Die Referenztexte werden dabei exakt, ohne Transformation wiedergegeben. Im Falle von Anspielungen findet hingegen eine sog. tiefenstrukturelle Referenz statt. Der Referenztext erscheint hier in der Regel nicht oder nur in einer modifizierten Form, wobei eine unveränderte Übernahme des Referenztextes auch möglich ist. Letzteres eröffnet wiederum die Problematik, inwieweit Anspielungen von Zitaten abgegrenzt werden können. Das gilt vor allem in dem Falle, wenn das übernommene Textmaterial von relativ geringem Umfang ist, z. B. in Überschriften. Sowohl bei Zitaten als auch bei Anspielungen werden bestimmte Marker eingesetzt, die Intertextualität signalisieren (können) (BURGER 2005:97-112). Bei den Zitaten sind es in erster Linie typografische Elemente, etwa die Verwendung von Kursivsatz oder einer vom Haupttext abweichenden Schriftart, der Einsatz von Textbegrenzungssignalen (hier: Anführungszeichen) oder die Angabe der Verfasser/innen des Referenztextes. Anspielungen zeichnen sich im Gegensatz dazu grundsätzlich durch die Abwesenheit solcher expliziten Marker aus und die Erkennung von intertextuellen Bezügen wird den Leser/innen überlassen. Die entscheidende Rolle spielen hier die Kreativität und die Vorkenntnisse der Rezipient/innen, die aktiviert werden müssen, um die Absicht der Verfasser/innen zu dekodieren.

\section{Zur kognitiven Leistung von Anspielungen}

Von dem Konzept ausgehend, dass Intertextualität durch den bewussten Einsatz fremder Texte im eigenen Text entsteht, ist hier anzunehmen, dass im Falle von Anspielungen der Verfasser/innen die Leser/innen absichtlich in ein kreatives Spiel einbezogen werden. Dieses beruht auf der menschlichen Assoziationskraft, die aktiviert werden muss, um die Absicht der Autor/innen zu ermitteln. Das bedeutet zugleich, dass Anspielungen erst dann entstehen, wenn die von den Verfasser/innen bei den Leser/innen erwarteten Vorkenntnisse tatsächlich vorhanden sind und die zutreffenden Textteile als Marker von Intertextualität erkannt werden. Es reicht dabei das bloße lexikalische Wissen nicht aus; die Leser/innen sollten über einen gewissen Grad an kombinatorischer Fantasie verfügen. Das heißt, Anspielungen eröffnen das Tor zu einem intellektuellen Spiel. Aus diesem Grund ist auch bei den Autor/innen eine besonders gute Kenntnis des Zielpublikums erforderlich, d.h. die Produzent/innen und Rezipient/innen sollten zumindest teilweise über das gleiche Vorwissen 
verfügen. Dieser Tatsache kommt vor allem bei Journalist/innen eine grundlegende Rolle zu. Durch Umfragen und regelmäßige Leseranalysen können über die jeweilige Zielgruppe umfassende Angaben ermittelt werden, die u.a. als Ausgangsbasis bei der Profilierung der einzelnen Rubriken eingesetzt werden können. So ist es kein Zufall, dass z. B. Anspielungen in Überschriften in erster Linie in Kommentaren, Feuilletons oder in Reportagen, also in komplexeren Textsorten vorkommen, während die für die schnelle Lektüre bestimmten Kurzberichte und Schlagzeilen in der Regel informativ und prägnant formulierte, direkt an die Leser/innen adressierte Titel haben.

Unabhängig davon, ob Anspielungen in literarischen oder nicht-literarischen Texten vorkommen, sind sie Mittel eines interaktiven Dialogs zwischen den Textproduzent/innen und Textrezipient/innen. Eine neue Dimension dieser Interaktion öffnet sich hingegen, wenn Anspielungen als Instrument der Abgrenzung verstanden werden, und zwar zwischen den Journalist/innen als Expert/innen und den Leser/innen als Lai/innen. In diesem Sinne versuchen die Journalist/innen durch den bewussten Einsatz von Anspielungen eine gewisse Distanz zu den Leser/innen aufzubauen, die eventuell auch als Ausdruck seiner Intellektualität bzw. seines Expertentums betrachtet werden kann. Die Textrezipient/innen werden hier als Konsument/innen betrachtet, die vom Wissen ausgeschlossen sind. Das birgt jedoch die Gefahr, dass der Dialog erfolglos bleibt und die Anspielung zwecklos oder missverstanden wird (vgl. HeUMANN 2015:43).

Die oben beschriebenen Eigenschaften machen Anspielungen zu einem beliebten und häufig eingesetzten Instrument der journalistischen Arbeit. Da sie unerwartet auftreten, verfügen sie über einen hohen Originalitätswert. Als solche können sie wesentlich zur Vermarktung des Produktes, in diesem Falle des Zeitungsartikels beitragen, insbesondere, wenn Anspielungen nicht im Haupttext, sondern im Titel vorkommen. Somit werden bei den Leser/innen bereits beim ersten Kontakt mit dem Artikel kognitive Operationen vollzogen. Das heißt, durch die Assoziationskraft werden vorhandene Wissensbestände aktiviert und durch den Vergleich des Bekannten mit dem Neuen erfolgt das Verständnis und neues Wissen wird produziert.

Anspielungen funktionieren somit als halbfertige Produkte, die erst im erfolgreichen Dialog zwischen Verfasser/innen und Leser/innen vollständig werden. Es ist jedoch hinzuzufügen, dass sie zugleich als Einweg-Formulierungen gelten, denn ihr Informationswert ist meist sehr gering. Die große Leistung liegt vorwiegend in deren Originalitätswert, nützliche Informationen über den KoText vermitteln sie dagegen kaum. Durch die wiederholte Verwendung von 
Anspielungen kann es dabei zu einer Verfestigung von Anspielungen der Sprachbenutzer/innen kommen. So können sog. Anspielungsmuster - in der Tat neue Sprachfloskeln - entstehen, wobei die ursprünglichen Formulierungen und somit die Quintessenz von Anspielungen verloren gehen (vgl. WILSS 1989:4). Die Journalist/innen können zwar durch die wiederkehrende Neukonfiguration ein theoretisch endloses Instrumentarium von Anspielungen schaffen, ${ }^{2}$ die regelmäßig wiederkehrenden Formulierungen - auch mit Modifikationen - können jedoch den Überraschungseffekt erheblich schwächen. Vor allem erweisen sich die übertriebenen Konstruktionen in Überschriften als selbstzweckhaft, in denen ein thematischer Bezug zwischen Titel und Ko-Text kaum mehr besteht.

\section{Empirische Untersuchung}

\subsection{Typologie nach Quellenbereichen}

Obwohl das Untersuchungskorpus einen Ausschnitt der aktuellen Einwanderungsdebatte darstellt und somit in einen relativ spezifischen Kontext eingebettet ist, finden bei den Quellenbereichen diese Besonderheiten keinen signifikanten Niederschlag. Die Mehrheit der ermittelten Anspielungen lässt sich der Domäne Kunst zuordnen (insgesamt 38 Einträge). Es sind hierbei die Subdomänen Musik (3), Literatur (20) und Film (15) vertreten. Ein Eintrag gehört zur Domäne Gastronomie (vgl. Bsp. 27) und 14 Einträge gelten als Phraseologismen (Sprichwörter, Alltagsfloskeln). Besondere Bedeutung kommt einer Formulierung zu, die in den untersuchten Zeitungsartikeln in acht Varianten vorkommt. Es handelt sich um den Satz Wir schaffen das, den die Bundeskanzlerin Angela Merkel auf einer Pressekonferenz am 31. August 2015 äußerte und der seitdem den gesamten deutschen Migrantionsdiskurs grundlegend geprägt hat (als Vertreter der Domäne Politik). Neben Willkommenskultur drückt nämlich gerade dieser europaweit berühmt gewordene Satz die - zumindest bisherige - offizielle Linie der Einwanderungspolitik der Bundesregierung aus. Die Tatsache, dass selbst in diesem kleinen Korpus in einer relativ hohen Anzahl Variationen der ursprünglichen Formulierung als Anspielungen vorkommen, deutet zugleich mit Blick auf die bundesdeutsche Ausländerpolitik auf die besondere Meinungsvielfalt hin.

2 Vgl. das Prinzip der variierenden Wiederholung bei BESCH (1989:5). 
(1) Wir schaffen das, wenn ...

[FAZ, 19.09.2015: Die Realisierung der durch die Koalition vereinbarten Änderungen im Asylsystem hängt von mehreren Maßnahmen ab] $]^{3}$

(2) Was wir schaffen müssen

[FAZ, 02.08.2016: Aufgaben der Bundesregierung, um die Flüchtlingskrise bzw. den Terror zu besiegen]

(3) Wir schaffen das nicht mehr

[FAZ, 21.10.2015: Gemeinden in NRW verlangen Unterstützung von der Bundesregierung zur Bewältigung der Welle von Asylbewerber/innen]

(4) Die schaffen sie

[FAZ, 19.10.2015: Widerstand innerhalb der CDU gegen die Flüchtlingspolitik der Bundeskanzlerin]

(5) Schaffen wir das?

[FAZ, 02.08.2016: Die polarisierende Flüchtlingspolitik Deutschlands bietet Nährboden für Extremismus und Populismus]

(6) Es dürfte zu schaffen sein

[SZ, 11.11.2015: Einsatz von Flüchtlingen auf dem deutschen Arbeitsmarkt ist möglich, jedoch unter besonderen Bedingungen]

(7) Ich schaffe das

[SZ, 12.02.2016: Wegen ihrer Flüchtlingspolitik kämpft die Bundeskanzlerin um ihr politisches Überleben]

(8) Was zu schaffen ist

[SZ, 29.03.2016: Konkrete Maßnahmen zur Lösung der Flüchtlingskrise in der Bundesrepublik]

Aus den obigen Beispielen wird ersichtlich, dass die einzelnen Überschriften ihren Kerngedanken in der ursprünglichen Äußerung von Angela Merkel haben. Sie thematisieren jedoch Aspekte, die entweder als Folgen der optimistischen Einstellung der Bundeskanzlerin oder als Voraussetzungen zu betrachten sind, damit es Deutschland tatsächlich ,schafft ${ }^{\star}$. Als Folgen gelten u.a. die starke Polarisierung der Gesellschaft sowie die innere Opposition in der Koalition (Bsp. 4, 5, 7). Auf der anderen Seite sollte die Regierung, worauf die Akteur/innen in den entsprechenden Artikeln hinweisen, auf manchen Gebieten - z. B. auf dem Arbeitsmarkt und im Rechtssystem - einige Maßnahmen

3 Zusammenfassungen durch A.M.; die einzelnen Texte sind im Internet verfügbar. 
treffen, die dazu erforderlich sind, dass die Flüchtlingswelle überhaupt bewältigt wird (Bsp. 1, 2, 6, 8). Der Rückgriff auf die ursprüngliche Formulierung zeichnet sich einerseits als kreatives journalistisches Instrument aus, um das Interesse der Leser/innen zu wecken. Andererseits liefern selbst diese wenigen Belege ein eklatantes Beispiel dafür, wie anfangs kreativ wirkende Anspielungen sich rasch zu Alltagsfloskeln entwickeln und ihren Originalitätseffekt verlieren (können). Die Karriere von Wir schaffen das beendete die Bundeskanzlerin selbst, indem sie sich im September 2016 von ihrem berühmtesten Satz zur Flüchtlingskrise eindeutig distanzierte:

Er ist Ausdruck einer Haltung, wie sie sicher viele aus ihrem beruflichen und privaten Leben kennen. Manchmal denke ich aber auch, dass dieser Satz etwas überhöht wird, dass zu viel in ihn geheimnist wird. So viel, dass ich ihn am liebsten kaum noch wiederholen mag, ist er doch zu einer Art schlichtem Motto, fast zu einer Leerformel geworden. ${ }^{4}$

Es ist darüber hinaus anzumerken, dass der Satz der Bundeskanzlerin in anderen, hier nicht untersuchten Medien in weiteren Varianten vorkommt. Das bietet zugleich die Basis für eine komparative Analyse, welche Anspielungen durch solche Modifikationen des Prätextes entstehen, die sich auf das gleiche Ereignis beziehen (vgl. auch Bsp. 29 und 31).

\subsection{Typologie nach Art der Bezugnahme auf den Prätext}

Es handelt sich im Folgenden um die Qualität der Markierung der Beziehung zwischen Anspielung und Prätext. Volle Identität, d.h. Einbettung des Prätextes ohne Modifizierung - also die Übernahme des referierten Titels -, kommt in 13 Einträgen vor. Mit zehn Anspielungen ist hier die Domäne Kunst vertreten, darunter die Subdomänen Literatur, Film und Musik:

Kunst generell:

(9) Neue Sachlichkeit

[FAZ, 15.01.2016: Nach einem konfliktreichen Jahr wollen die Berliner SPD und CDU konstruktiv in das neue Jahr gehen]

\section{Subdomäne Literatur:}

(10) Inseln im Strom

[FAZ, 15.02.2016: Kritik der Visegrád-Staaten an der deutschen Flüchtlingspolitik]

WirtschaftsWoche, 17.09.2016, URL: http://www.wiwo.de/politik/deutschland/fluecht lingskrise-angela-merkel-will-wir-schaffen-das-nicht-wiederholen-/14556964.html (23.09.2017). 
(11) Mutter Courage

[FAZ, 06.06.2016: Anne Hidalgo, die Bürgermeisterin von Paris - eine starke Frau im Schatten des Terrorismus]

(12) Die Verwandlung

[SZ, 28.11.2015: Der frühere Konservative Alexander Gauland als neuer ,Scharfmacher der AfD]

(13) Mutter Courage

[SZ, 17.05.2016: Porträt von Anne Hidalgo, der Bürgermeisterin von Paris]

Subdomäne Musik:

(14) Sing, mei Sachse, sing!

[FAZ, 21.07.2015: Die AfD in Sachsen an der Grenze zum Rechtsextremismus]

Subdomäne Film:

(15) Das Leben der Anderen

[FAZ, 19.10.2015: Vorstellungen über das Leben und die Träume der Flüchtlinge]

(16) Der Schatz im Silbersee

[FAZ, 25.10.2015: Durch erleichterte Hochschulzugänge für Flüchtlinge senkt das Bildungsniveau rapide]

(17) Kebab Connection

[FAZ, 16.03.2016: Endlich Zusammenarbeit zwischen Griechenland und der Türkei im Rahmen der NATO]

(18) Türkisch für Anfänger

[SZ, 19.02.2016: Tücken der Einigung über die Rücknahme von Flüchtlingen zwischen der Türkei und der EU]

Partielle Identität kommt hingegen in 51 Fällen vor; das sind Anspielungen, bei denen die referierten Prätexte mehr oder weniger modifiziert wurden. Die einzelnen Modifikationstypen lassen sich auf die Transformationstechniken der Rhetorik zurückführen (vgl. WILSS 1989:144f.). Demnach werden hier Anspielungen mit partieller Identität den folgenden Gruppen zugeordnet:

\section{Substitution:}

(19) Der gute Mensch von Sizilien

[FAZ, 24.04.2015: Der Bürgermeister von Palermo fördert einen humanitären Korridor für Flüchtlinge]

(20) Die Rückkehr des Thomas de Maizière

[FAZ, 12.12.2015: Nach vielen Konflikten ist die Position des Bundesinnenministers wieder stabil] 


\section{Hinzufügung:}

(21) Szenen einer kaputten Ehe

[SZ, 05.06.2016: Streit zwischen der CDU und CSU wegen der Flüchtlingsfrage]

(22) Wir schaffen das nicht

[FAZ, 13.10.2015: Gemeinden verlangen Unterstützung zur Bewältigung der Welle von Asylbewerber/innen]

\section{Kürzung:}

(23) Tröglitz ist nicht überall

[FAZ, 08.04.2015: Deutschland ist generell nicht fremdenfeindlich; als Reaktion auf die Worte des Ministerpräsidenten von Sachsen-Anhalt]

\section{Permutation:}

(24) Schaffen wir das?

[FAZ, 2.08.2016: Die polarisierende Flüchtlingspolitik Deutschlands bietet Nährboden für Extremismus und Populismus]

Wenn die beiden Strategien miteinander verglichen werden, lässt sich feststellen, dass ein höheres Niveau an Identität mit dem Prätext zugleich eine höhere Komplexitätsstufe beim Dekodieren der Anspielung bedeutet. Gemeint ist hier, dass nicht modifizierte Überschriften - Anspielungen mit voller Identität - auf den ersten Blick keinen unmittelbaren Zusammenhang mit dem Ko-Text aufweisen. Hier wird die Aufgabe, das Rätsel der Überschrift zu dekodieren, vollständig dem Leser weitergegeben, d.h., die Rolle eines entsprechenden Vorwissens ist gerade bei diesem Typ am größten. Bei Anspielungen mit partieller Identität findet jedoch bereits auf Seiten der Textverfasser/innen eine Modifizierung statt, die die Leser/innen bei der Entschlüsselung der Anspielung eventuell unterstützen kann. Das gilt für Überschriften, in denen ein wesentliches Element - in der Regel ein Substantiv - durch ein anderes ersetzt wurde. Das Ersatzelement liegt in der Regel dem Inhalt des Ko-Textes näher, indem es weniger das Aktivieren des Vorwissens bei den Leser/innen verlangt, sondern vielmehr eine eindeutige Beziehung zwischen Anspielung und Prätext herstellt. Diese Ersatzelemente können als Schlüsselwörter betrachtet werden, deren Funktion im nächsten Abschnitt ausführlich erläutert wird. 


\section{Diskursspezifischer Wortschatz als Basis für modifizierte Über- schriften}

Wie die hier aufgeführten Beispiele illustrieren, liefern die einzelnen Überschriften nicht unbedingt viele Informationen über den Inhalt des Ko-Textes. Inwieweit sie überhaupt im Zusammenhang mit dem Migrationsdiskurs stehen, lässt sich oft erst durch die Interpretation des jeweiligen Zeitungsartikels ermitteln. Als hilfreich zeigt sich jedoch eine Analyse der Leitbegriffe in Überschriften. Auf diese Weise kann ein Inventar jener Ausdrücke ermittelt werden, die einen Diskurszusammenhang herstellen, die also miteinander den jeweiligen Diskurs konstituieren. Das heißt, den eingeweihten Leser/innen mit entsprechenden Vorkenntnissen wird bereits anhand der vorläufigen Lektüre der Überschriften schnell klar, dass der jeweils nachfolgende Artikel das Thema Flüchtlinge behandelt und somit zum Flüchtlingsdiskurs gehört. Bezüglich der Zugehörigkeit zur Einwanderungsdebatte wird hier zwischen primärem und sekundärem Wortschatz unterschieden. Dem primären Wortschatz werden diejenigen Ausdrücke zugeordnet, die dem sog. allgemeinen Interaktionsvokabular angehören (vgl. KLEIN 1989:7) und langfristig mit der Einwanderungsdebatte verbunden werden:

(25) Alles eine Frage der Quote

[FAZ, 12.05.2015: Die EU plant die Einführung von Flüchtlingsquoten in den Mitgliedstaaten]

(26) Asyl ungarischer Art

[FAZ, 04.09.2015: Tricks der ungarischen Polizei, Flüchtlinge in Aufnahmelager zu schleusen]

(27) Der Terrorist, der dann doch keiner war

[FAZ, 18.09.2015: Kritik an der polizeilichen Gewalt gegen Flüchtlinge in Ungarn]

(28) Muslime, nein danke

[FAZ, 04.03.2016: Die Slowakei will keine muslimischen Flüchtlinge aufnehmen]

(29) Dame, König, Asyl

[SZ, 28.07.2015: Flüchtlingspolitik in Baden-Württemberg]

(30) Muslime unerwünscht

[SZ, 21.08.2015: Widerstand der Slowakei gegen die Aufnahme von muslimischen Flüchtlingen] 
Die hervorgehobenen Ausdrücke bilden einen festen Bestandteil des migrationsspezifischen Wortschatzes. Durch deren Identifizierung als diskursrelevante Begriffe werden die zugehörigen Titel entsprechend bewertet und somit wird auch weniger attraktiven Überschriften bzw. Artikeln eine höhere Bedeutung zugeschrieben.

Zum sekundären Wortschatz gehören hingegen Ausdrücke mit einer eher kurzfristigen Brisanz. Diese sind eng mit bestimmten Phasen des Diskurses verbunden, worin sie durch Assoziationen der Sprachbenutzer/innen in konkreten Situationen ein aktualisiertes semantisches Profil erhalten. Es handelt sich dabei in erster Linie um Akteur/innen, die in einem gewissen Zeitraum in dem Flüchtlingsdiskurs eine relevante Rolle gespielt haben. Neben Personen, die hier als Akteur/innen auftreten, gelten auch einige Institutionen (z. B. BAMF, EU-Kommission), Orte (z. B. Calais, Tröglitz, Idomeni) oder Gegenstände (z. B. Zaun, Gesundheitskarte) als Entitäten, denen im thematisierten Diskurs eine besondere Rolle zukommt.

(31) Der gute Mensch von Sizilien (FAZ, 24.04.2015)

[Sizilien ist das erste Ziel von Flüchtlingen auf der Mittelmeerroute]

(32) Die Rückkehr des Thomas de Maizière (FAZ, 12.12.2015)

[der Bundesinnenminister ist für die Lösung des Flüchtlingsproblems primär zuständig]

(33) Ein Geisterschiff wird kommen (FAZ, 17.01.2015)

[Menschenschmuggler schmuggeln Flüchtlinge mit Schiffen ohne Besatzung]

Dadurch, dass die in den obigen Beispielen hervorgehobenen alltagssprachlichen Ausdrücke im Kontext der Einwanderungsdebatte konzentriert vorkommen, werden diese durch die Sprachbenutzer/innen mit dem Flüchtlingsdiskurs assoziiert. Da es sich dabei nicht um solche, im Inventar der politischen Sprache verfestigten Ausdrücke wie Asyl handelt, führt durch die Dynamik der Ereignisse bzw. des Diskurses ein Verlust an Relevanz gleichzeitig auch zu einer Aktualisierung von deren semantischen Profilen und evtl. zu deren Verschwinden aus dem sekundären Wortschatz des Flüchtlingsdiskurses.

\section{Fazit}

Anspielungen gelten als feste Bestandteile des journalistischen Kanons; sie kommen daher in Pressetexten besonders häufig vor. Sie stellen ein Instrument der indirekten Kommunikation zwischen Verfasser/innen und Leser/innen dar und erfüllen folglich eine doppelte Funktion. Einerseits treten sie als stilistische 
Mittel auf, um die Aufmerksamkeit der Leserschaft zu fesseln bzw. zu steuern, um auf diese Weise das Produkt - den Zeitungsartikel - an die Leser/innen zu bringen. Auf der anderen Seite haben Anspielungen eine sinnkonstituierende Funktion. Sie setzen Assoziationskraft und entsprechende Vorkenntnisse bei den Leser/innen voraus, die aktiviert und in die Dekodierung der verdeckten Botschaft einbezogen werden müssen, damit die Anspielung überhaupt verstanden wird und wirkt.

Diese spielerische Natur von Anspielungen ermöglicht es auch den Verfasser/innen, ihre Meinung über Themen zu äußern, die in der Gesellschaft bzw. beim Zielpublikum aus verschiedenen Gründen evtl. als ,heiß` eingestuft werden. Anspielungen bieten Raum für Ironie, sanften Humor, aber ebenso für scharfe Kritik. Da sie auf die Intellektualität der Leser/innen bauen, können die Verfasser/innen gezielt auf Quellendomänen zurückgreifen, die das Verständnis des Textinhalts fördern. Einen solchen Quellenbereich verkörpert die Literatur. Wie auch die hier aufgeführten Belege zeigen, sind Anspielungen auf allgemein bekannte literarische Werke besonders beliebt, da gerade in dieser Relation bei den Leser/innen einige Vorkenntnisse vorausgesetzt werden können.

Inwieweit Anspielungen mit voller oder partieller Identität eingesetzt werden, hängt grundsätzlich von den Absichten der jeweiligen Verfasser/innen ab. Im Falle von Formulierungen mit voller Identität sind die Leser/innen ausschließlich an das vom Journalisten eingesetzte Konstrukt angewiesen, wodurch zur Interpretation der Phantasie mehr Raum gelassen wird. Es werden hier unterschiedliche Quellenbereiche unmodifiziert referiert, der Wiedererkennungseffekt ist daher entsprechend größer. Die hohe Anzahl der Anspielungen, die zum Quellenbereich Kunst gehören, bestätigt die Annahme, dass in erster Linie solche Quellenbereiche bzw. Prätexte bevorzugt werden, die bei den Leser/innen entsprechende allgemeine Kenntnisse u.a. auf dem Gebiet der Literatur oder der Filmkunst voraussetzen.

Bei Anspielungen mit partieller Identität erlebt man den kreativen Moment der Entschlüsselung nicht in seiner Totalität, da die Leser/innen durch die modifizierte Überschrift bereits bei der ersten Begegnung mit dem Artikel einen Hinweis zum Textinhalt bekommen. Das gilt vor allem dann, wenn das neue Element in der Überschrift einen direkten Zusammenhang mit dem Textthema herstellt. In dem hier untersuchten Korpus sind es diejenigen Ausdrücke (vgl. Bsp. 26-31), die als Schlüsselwörter zum Wortschatz des Einwanderungsdiskurses gehören. Da diese aus der regelmäßigen themenspezifischen Berichterstattung allgemein bekannt sind, beschleunigen sie auch wesentlich das Verständnis der Anspielung. 
Der im vorliegenden Beitrag thematisierte Flüchtlingsdiskurs gilt als ein empfindliches Thema. Die vielfältigen Aspekte dieser Problematik finden in einer besonders lebhaften Debatte ihren Niederschlag. In der gegenwärtigen Mediengesellschaft steht auf allen Gebieten (politische) Korrektheit an erster Stelle, insbesondere in der politischen Berichterstattung. Anspielungen zeigen sich daher als besonders geeignete Mittel, unterschiedliche Meinungen und Standpunkte nicht direkt auszusprechen, sondern in Form von verschlüsselten Botschaften zu übermitteln. Missverständnis oder Nicht-Verständnis sind zwar auch ein Teil des Spiels zwischen Verfasser/innen und Leser/innen, aber Anspielungen als zufällige, nicht systematische Bildungen kommt beim Schaffen von neuem Wissen - ähnlich wie Metaphern - eine grundlegende Bedeutung zu.

\section{Literatur}

AdAMZIK, KirSTEN (2004): Sprache. Wege zum Verstehen. 2., überarb. Aufl. Tübingen. ANDROUTSOPOULOS, JANNIS (1997): Intertextualität in jugendlichen Textsorten. In: KLEIN, JOSEF / FIX, UlLA (eds.): Textbeziehungen. Linguistische und literaturwissenschaftliche Beiträge zur Intertextualität. Tübingen, 339-372.

Barthes, Roland (2000): Der Tod des Autors. Aus dem Französichen von Matias Martinez. In: JANNidis, Fotis / LAUER, GERHARD / MARTineZ, MATIAS / WinKo, SimONE (eds.): Texte zur Theorie der Autorschaft. Stuttgart, 185-197.

BARTHES, Roland (1981): The Theory of the Text. In: Young, RoBert (ed.): Untying the Text: A Post-Structuralist Reader. Boston, 31-47.

BESCH, ElmAR (1989): Wiederholung und Variation. Untersuchung ihrer stilistischen Funktionen in der deutschen Gegenwartssprache. Frankfurt a. M.

BÖKE, KARIN (2002): Wenn ein ,Strom “ zur ,Flut“ wird. Diskurslinguistische Metaphernanalyse am Beispiel der Gastarbeiter- und Asyldiskussion in Deutschland und Österreich. In: PANAGL, Oswald / STÜRMer, Horst (eds.): Politische Konzepte und verbale Strategien. Frankfurt a. M., 265-286.

BÖKE, KARIN (1997): Die „Invasion“ aus den „Armenhäusern Europas“. Metaphern im Einwanderungsdiskurs. In: Jung, MatTHIAS (ed.): Die Sprache des Migrationsdiskurses. Das Reden über ,Ausländer “ in Medien, Politik und Alltag. Opladen, 164-193. BöKe, Karin / Jung, Matthias / Wengeler, Martin (eds.) (1996): Öffentlicher Sprachgebrauch. Praktische, theoretische und historische Perspektiven. Georg Stötzel zum 60. Geburtstag gewidmet. Opladen.

BöKe, KARIN / LiedtKe, Frank / Wengeler, MARTin (eds.) (1996): Politische Leitvokabeln in der Adenauer-Ära. Berlin / New York. 
BroICH, UlRICH (1985): Formen der Markierung von Intertextualität. In: BROICH, UlRICH / PFISTER, MANFRED (eds.): Intertextualität. Formen, Funktionen, anglistische Fallstudien. Tübingen, 31-47.

Burger, HARALD ( $\left.{ }^{3} 2005\right)$ : Mediensprache. Eine Einführung in Sprache und Kommunikationsformen der Massenmedien. Aufl. Berlin / New York.

De Beaugrande, Robert Alain / Dressler, WolfGang (1981): Einführung in die Textlinguistik. Tübingen.

GARDT, ANDREAS (2007): Diskursanalyse - Aktueller theoretischer Ort und methodische Möglichkeiten. In: WARNKE, INGO H. (ed.): Diskurslinguistik nach Foucault. Theorie und Gegenstände. Berlin / New York, 27-52.

Heumann, Anja (2015): Spiel und Anspielung. Der literarische Kanon in journalistischen Texten. Würzburg (=Epistemata Literaturwissenschaft 824).

Holthuis, SuSANNE (1993): Intertextualität. Aspekte einer rezeptionsorientierten Konzeption. Tübingen (Stauffenburg-Colloquium 28).

Jung, MatThias / NiEhr, Thomas / BöKe, KARIN / DorenBeck, NiLS (2000): Ausländer und Migranten im Spiegel der Presse. Ein diskurshistorisches Wörterbuch zur Einwanderung seit 1945. Wiesbaden.

Klein, Josef (ed.) (1989): Politische Semantik. Bedeutungsanalytische und Sprachkritische Beiträge zur politischen Sprachverwendung. Wiesbaden.

KristeVA, Julia (1969): Sèméiotiké: Recherches pour une sémanalyse. Paris.

LAKOFF, GEORGE/JOHnSON, MARK (2007): Leben in Metaphern. Konstruktion und Gebrauch von Sprachbildern. Heidelberg.

LATOUR, BRUNO / RoßLER, GUSTAV (2007): Eine neue Soziologie für eine neue Gesellschaft. Einführung in die Akteur-Netzwerk-Theorie. Frankfurt a. M.

LeIDECKer, Melanie (2015): „Das ist die Topgeschichte des Tages!“ AufmacherArtikel deutscher Tageszeitungen im Vergleich. Köln.

LEIFELD, PHILIP (2009). Die Untersuchung von Diskursnetzwerken mit dem Discourse Network Analyzer (DNA). In: SCHNEIDER, VolKER / JANNING, FrAnK / LEIFELD, PHILIP / Malang, Thomas (eds.): Politiknetzwerke, Modelle, Anwendungen und Visualisierungen. Wiesbaden, 391-404.

NieHr, ThOMAs (2014a): Einführung in die linguistische Diskursanalyse. Darmstadt.

NieHr, Thomas (2014b): Einführung in die Politolinguistik. Gegenstände und Methoden. Stuttgart.

NIEHR, THOMAS / BÖKE, KARIN (eds.) (2000): Einwanderungsdiskurse. Vergleichende diskurslinguistische Studien. Wiesbaden.

PetÖFI, JÁnOS S. / Olivi, TERRY (1988): Schöpferische Textinterpretation. Einige Aspekte der Intertextualität. In: PETÖFI, JÁNOS S. / OLIVI, TERRY (eds.): Von der verbalen Konstitution zur symbolischen Bedeutung. Hamburg, 335-350. 
PFISTER, MANFRED (1985): Konzepte der Intertextualität. In: BROICH, UlRICH / PFISTER, MANFRED (eds.): Intertextualität. Formen, Funktionen, anglistische Fallstudien. Tübingen, 1-30.

REITER, MARKUS (2006): Überschrift, Vorspann, Bildunterschrift. Konstanz.

Schneider, Volker / JANning, Frank / Leifeld, Philip / Malang, Thomas (2009): Politiknetzwerke. Modelle, Anwendungen und Visualisierungen. Wiesbaden.

SVEnsson, ARnOld (1984): Anspielung und Stereotyp. Eine linguistische Untersuchung des politischen Sprachgebrauchs am Beispiel der SPD. Opladen.

WENGELER, MARTIN (2003): Topos und Diskurs: Begründung einer argumentationsanalytischen Methode und ihre Anwendung auf den Migrationsdiskurs (1960-1985). Berlin / New York.

Wilss, Wolfram (1989): Anspielungen. Zur Manifestation von Kreativität und Routine in der Sprachverwendung. Tübingen. 